\title{
Conditions for effective smart learning environments
}

Rob Koper

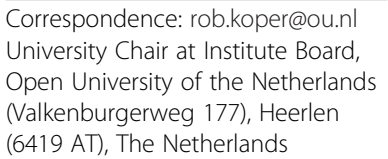

Correspondence: rob.koper@ou.nl University Chair at Institute Board, Open University of the Netherlands (Valkenburgerweg 177), Heerlen (6419 AT), The Netherlands

\begin{abstract}
Smart learning environments (SLEs) are defined in this paper as physical environments that are enriched with digital, context-aware and adaptive devices, to promote better and faster learning. In order to identify the requirements for 'better and faster learning', the idea of Human Learning Interfaces (HLI) is presented, i.e. the set of learning related interaction mechanisms that humans expose to the outside world that can be used to control, stimulate and facilitate their learning processes. It is assumed that humans have and use these HLIs for all types of learning, and that others, such as parents, teachers, friends, and digital devices can interact with the interface to help a person to learn something. Three basic HLIs are identified that represent three distinct types of learning: learning to deal with new situations (identification), learning to behave in a social group (socialization) and learning by creating something (creation). These three HLIs involve a change in cognitive representations and behavior. Performance can be increased using the practice HLI, and meta-cognitive development is supported by the reflection HLI. This analysis of HLIs is used to identify the conditions for the development of effective smart learning environments and a research agenda for SLES.
\end{abstract}

Keywords: Human learning interfaces; Smart learning environments; Research agenda

\section{Introduction}

Why should we develop smart(er) learning environments? For me, the answer is to promote better and faster learning. Every step forward from existing learning environments towards these smarter learning environments is an improvement that is essential for further human, economical and cultural development. This is a big challenge. In this paper I will provide a contribution towards this ambition by exploring the conditions that should be met by smart learning environments (SLEs) in order to stimulate better and faster learning. For this purpose I will introduce a new theoretical concept, named Human Learning Interfaces (HLIs), that can facilitate the research and development of SLEs. I define HLIs as the set of interaction mechanisms that humans expose to the outside world, and that can be used to control, stimulate and facilitate their learning processes. An interface is the communication facility that enables two or more systems to communicate with each other, i.e. facilitate the creation and interpretation of messages that elicit a (learning) response (see Griffin 2012, p.6). It is assumed that humans have and use these HLIs for all types of learning, and also others, such as 
parents, teachers, friends and digital devices can interact with the interface to help a person to learn something. For instance in education HLIs could be seen as the interfaces between the teacher and the learner: the teacher uses the interfaces of the learners to enable them to learn something. The interfaces describe functions for input and output: appropriate input for the senses of the learner to stimulate learning, observing (output) behaviors and providing (input) feedback and feedforward. HLIs are abstractions of very complex sensory, cognitive and behavioral processes and can be utilized in any situation to learn something, also by the person himself, or by elements in the environment the person is in, including digital devices.

In the following sections I will elaborate the idea of HLIs. First I will identify and define some core concepts, followed by the description of the HLIs. Then I will present the definition of Smart Learning Environments, providing a strong research challenge for the field. In the discussion and conclusion I will discuss some research issues related to HLIs and its use.

\section{Core concepts}

First I will inspect three core concepts that are needed for HLIs: learning environments, smart learning environments and learning.

\section{Learning environment}

The term Learning Environment is used in different contexts with different meanings (see e.g. Abualrub et al. 2013). In the glossary of education reform a good definition is provided ("Glossary" 2014): "Learning environment refers to the diverse physical locations, contexts, and cultures in which students learn." Examples are classrooms, workplaces, labs, museums, natural sites, means of transport, and home. Most learning environments are deliberately arranged or adapted to stimulate learning towards some learning objectives, e.g. by adding learning materials, tasks, tests, feedback and support. A learning environment can be arranged more or less generic, i.e. supports a smaller or wider set of learning activities and learning objectives. Especially in modern learning theories, the importance of embedding learning activities within authentic environments is emphasized (Vygotsky 1978; Brown et al. 1989), i.e. by solving real world problems in the context in which they most typically occur (Merrill 2002).

There is also a more restricted use of the term learning environment, namely as an abbreviation of 'digital' or 'virtual' learning environments. Using the previous definition digital learning environments are digitally represented locations, contexts and cultures in which students learn. Examples are serious games and virtual classrooms. One of the problems of a focus on digital learning environment is that it leaves implicit that a digital environment is always embedded in a physical environment. When using a digitally represented environment, you are still located in a physical environment, such as a living room, that can influence the learning processes and the cognitive representation of the learning environment, e.g. by influencing attention processes, state of arousal, memory cueing, concentration, encoding and recall. So, when considering digital learning environments, they should always be perceived as the addition of digital devices to the current physical environment of humans, influencing the total cognitive representation of their environment, and through this, their behavior and learning processes. The 
term 'digital device' is used here to represent the combination of some computer-based hardware, such as a smartphone, robot, smart watch, smart glasses, smart board and computer, and some dedicated software that provides the concrete functionality for the learning process. Digital device(s) can be more or less aware of the physical context in which they are used. Context-aware devices (Gross and Specht 2001), typically have sensors that enable them to 'know' something about the environment they are in, including some measurable aspects of the user. This information can be used to enrich or augment the physical environment and to provide guidance and feedback to the user.

Learning environments can be defined as the set of physical and digital locations, contexts and cultures in which students learn. Five typical cases of learning environments can be distinguished with respect to their relation to digital devices:

1. The zero case: there are no relevant physical or digital relevant stimuli in the environment of a person. The cognitive representations of the person can be formed rather independent of the outside world: thinking, dreaming, visualising something based on memory and creativity processes. In this case there is an internally stimulated representation of the learning environment.

2. The digital case: when the physical environment includes digital learning devices, but does not provide relevant non-digital stimuli to the user. For instance in a quiet study room when using a simulation program. The representation of the learning environment can dominantly be influenced by the digital device(s), e.g. by presenting a virtual reality world, a serious game, a virtual classroom or a (digital) book. The cognitive representations that are stimulated by the digital device can result in learning processes. In this case there is a digital stimulated representation of the learning environment.

3. The embedded case: the physical environment provides relevant stimuli to the user and the digital devices are adding, augmenting information to enrich the cognitive representation. In this case there is a combined, partly digital, partly physical stimulated representation of the learning environment.

4. The side-by-side case: the digital devices are added to a physical environment to support additional learning functions such as information, support, tests and feedback, but the digital devices are ignorant of the actual physical environment. All information about the physical environment should be added to the device by the user. For example when students are presented with tasks to execute in their physical environment, but they need to input the results to the digital device themselves. In this case the user's representation of the learning environment is fragmented: the physical parts and the digital parts.

5. The classical case: the physical environment provides relevant stimuli, and there are no additional digital relevant signals. This is 'old school' situation where humans are interacting and learning without the help of any digital device. In this case there is a representation of the learning environment by the user that is stimulated by the physical environment.

Note that in any of the representations mentioned in the cases, different people have different representations because they are selective in attention, represent stimuli in the 
context of interests and pre-knowledge, and add and delete information to fit a view which is comfortable to them. Behavior and learning are the result of these individually different representations and not directly the result of the stimuli provided. This is why observation of the behavior, feedback, feedforward and adaptations of interventions are important when you want someone to learn something, e.g. in a smart learning environment.

\section{Smart learning environments}

What are smart learning environments? The idea of smart learning environments fits in the tradition of adding the adjective 'smart' to various existing phenomena, such as smart phones, smart tv's, smart boards, smart lights and smart cities in order to identify a next step in its development or a new generation. So, from this perspective smart learning environments could be seen as learning environments that are considerably improved to promote better and faster learning. So what type of improvements makes a learning environment smart? First of all, when looking at the previous section about learning environments, I would say that only the case 'embedded' could be considered smart. The zero case and the classical case do not use digital devices, and an implicit aim of smart learning environments is to use ICT to improve the learning environments. The digital case and the side-by-side case represent most of the current computer-based learning solutions such as serious games, tutorials, drill and practice and tests. They require the user to concentrate on the digital device that is responsible for (parts of) the learning process and the device itself is ignorant of the physical environment the user is in. So, for me, the first set of requirements for SLEs are the following. an SLE is a learning environment in which:

1. one or more digital devices are added to the physical locations of the learner;

2. the digital devices are aware of the learners location, context and culture;

3. the digital devices add learning functions to the locations, context and culture, such as the provision of (augmented) information, assessments, remote collaboration, feedforward, feedback, etc.;

4. a digital devices are monitoring the progress of learners and provides appropriate information to relevant stakeholders.

So, an SLE is context-aware and adaptive to the individual learner's behavior. However, concentrating on these technical aspects does not automatically promote better and faster learning. I would even state that it is a loss of time when SLEs are build without having concrete notions about the improvement in learning one wants to accomplish. In the next section I will look at learning itself.

\section{Human learning}

Human learning has been studied extensively in various disciplines such as psychology, cognitive sciences, neurosciences, pedagogy and anthropology. Schunk (2012, p.3) defines learning as follows: "Learning is an enduring change in behavior, or in the capacity to behave in a given fashion, which results from practice or other forms of experience". This definition contains three criteria: learning involves change, learning endures over 
time, and learning occurs through experience and not by means of heredity for instance. In this section some of the highlights of learning research will be summarized that are relevant for HLIs.

The behavioristic approach to learning has concentrated on learning processes that change or strengthen the association between stimuli and behavioral responses. It considers the intermediate human cognitive processes as a black box that can not be known and is not relevant to know for the explanation of learning. The cognitive sciences have criticised and extended this view by opening up the black box by not only looking at stimuli and responses, but also at cognitive processes that mediate their relationship. Furthermore some learning processes could not be explained by behavioristic theories, notably the work of Bandura et al. $(1961,1963,1973)$ made clear that people could learn from observing the behavior of others, even without reinforcement. Also he elaborated the notion of self-regulation (Bandura 1991), the ability to take control over your own learning objectives, learning activities, learning environment and assessment of progress. The cognitive tradition, inspired by information processing technologies, asks questions such as: which learning related functions and processes are executed in the brain? Ohlsson (2011, p.29) states that the central elements of cognition are mental representations, and that differences in mental representations explain differences in behavior. Cognitive functions (perception, remembering, thinking, acting and learning) are implemented by processes that create, utilize and revise representations. The processes are leading to three streams of events that should be explained by a general cognitive theory: subjective experience, actions and utterances. Ohlsson (p.44) defines learning as a meta-function for cognitive change and he states that it is implausible that cognitive change can be explained by a single learning mechanism. He makes a distinction between monotonic and non-monotonic learning mechanisms. Monotonic mechanisms, such as associations, are governed by fixed, unchangeable rules and are predictable. Nonmonotonic learning mechanisms are complex learning mechanisms that are intrinsically hard to predict, such as weather forecasts. Non-monotonic learning mechanisms are cognitive processes that enable humans to suppress their experience and override its imperative for actions (p.21). An example are creative processes that are closely linked to learning, because in order to create something new, someone must change their cognitive representations.

Functions and processes are expected to run within a cognitive architecture. The most widely used architecture is the modal model, an adapted version of the Atkinson and Shiffrin model $(1968,1971)$. Figure 1 represents the modal model with the distinct memory stores positioned between input and output.

One interesting perspective on cognition is the dual process theory (Evans and Over 1996; Sloman 1996; Stanovich 1999, Stanovich 2004; Kahneman and Frederick 2005), there is evidence that humans utilize two (or more) different cognitive processes at the same time, often referred to as system 1 and system 2 , or type 1 and type 2 . Type 1 processes are associative, fast, automatic, needs low effort, have a large processing capacity and work on the basis of learned heuristics. Type 2 processes are rule-based, slow, have limited capacity because of limitations of working memory, is consciously controlled and needs a high level of mental effort (Evans 2009). The idea is that all stimuli that are beyond sensory memory are always processed by type 1 processes, leading to an immediate intention for a response. Type 2 processes 'controls' the response 


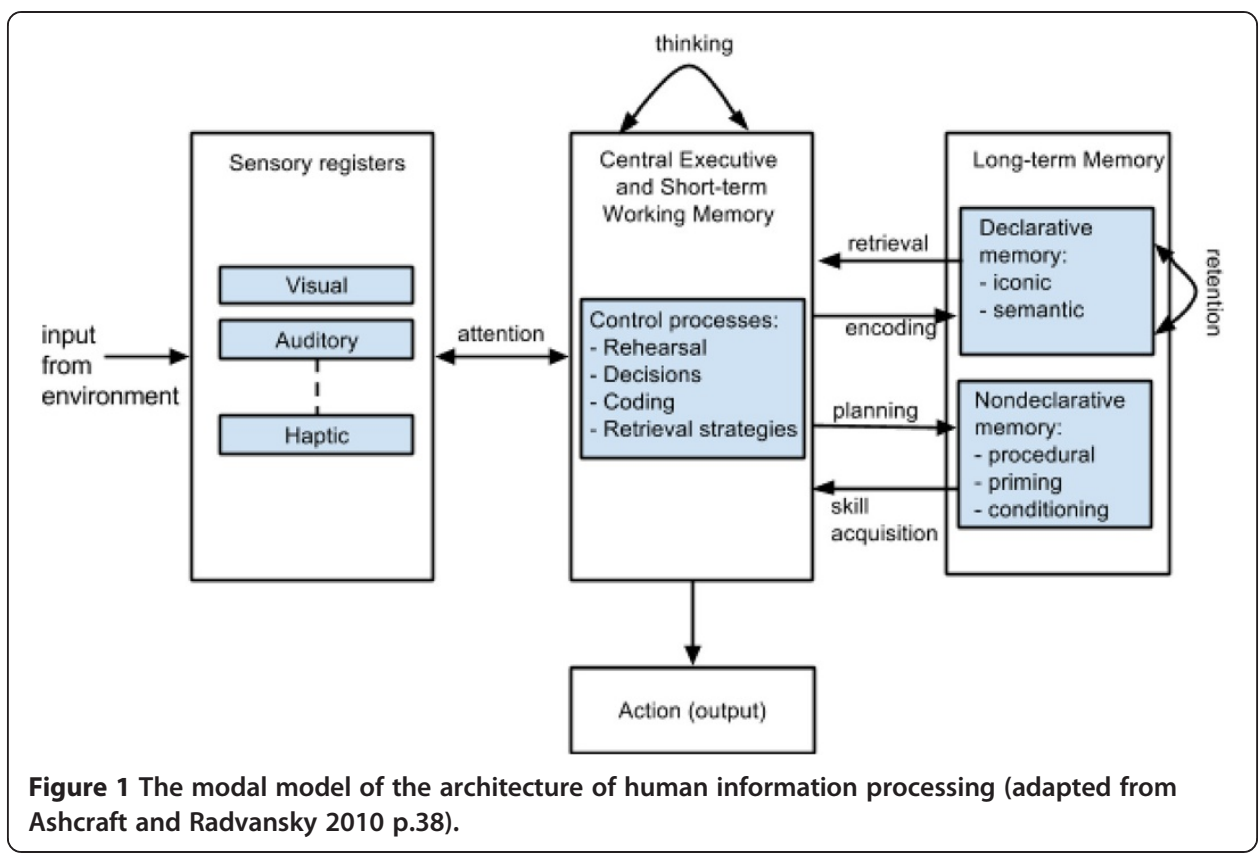

intentions and can rethink critically the assumed associative, often fake causal relationships used by type 1 processes. Type 2 is reflective and can approve the proposed action, cancel it, or change it. Because the activity of type 2 costs energy, people can be too lazy, too miser or too depleted (Baumeister et al. 1998) to control type 1 processes. Most of the actions of type 1, when appropriately practiced and learned could be very effective and efficient, but especially studies of Kahneman and Tversky (e.g., 1974, 1979) have shown that in more complex situations, especially when probabilities should be calculated or algorithms should be used, type 1 processes make the wrong guesses. This dual process theory comes close to another tradition of cognitive research: metacognition and especially self-regulation (e.g., Butler and Winne 1995). Metacognition refers to knowing what one knows and self-regulation is the process to govern activities in line with goals, environment and other priorities. A high level of self-regulation capabilities is needed for autonomous learning.

Besides cognition as a generic process, an important sub-discipline of social psychology should be mentioned: social-cognition, the field that is focussed on the relationship between human social behavior and cognition, including social learning as has been discussed earlier (see Carlston 2013). One of the concepts that are studied in social cognition is priming, a non-declarative long term memory function, the effect that the reaction to a stimulus is influenced by another stimulus (Meyer and Schvaneveldt 1971). Higgins et al. (1977) showed that introducing trait concepts in one task influences the trait related information in a non-related subsequent task. Many different kinds of priming have been found and can for instance be used to contextualise the interpretation of concepts to be learned. Of interest are also studies of socialization, i.e. the process in which individuals are learning the perceptions, norms, values and actions that are necessary to participate in a social groups such as family, work, peer groups, organisations. (e.g. Mayer 2013; Hafferty and Hafler 2011). 


\section{Human learning interfaces Generic model of HLIs}

Humans interface to the outside world through their senses (input) and their behavior (output). When designing an SLE, the environment must provide the right inputs and monitor the outputs in order to stimulate or facilitate learning processes. The use of these inputs and outputs can be generalized in a Human Learning Interface. In its most generic form it contains an intervention mechanism that provides the input for the senses, observations to monitor the behavior and physical state of the person, and observations to monitor changes in the physical environment. To control the learning towards certain learning objectives, it should be possible to specify a goal and to compare the observations with the goal to redirect the interventions (Figure 2).

A smart learning environment is identical to the physical environment of the user, with the addition of some digital devices that utilize the HLI (white box in Figure 2). In some situations the physical environment of the user is already arranged as a learning environment, such as a class, but this is not necessarily so. Digital devices can be added to any environment to create a learning experience. In social learning the digital devices also interact with other humans, e.g. to create a learning community. The core elements of the HLI will now be discussed.

\section{Interventions}

What type of interventions can be distinguished? I make a distinction between four core interventions for learning:

1. Ask a question to the learner. Asking questions invokes all kinds of cognitive processes. It invokes priming (questions offer a context), retrieval of information, it

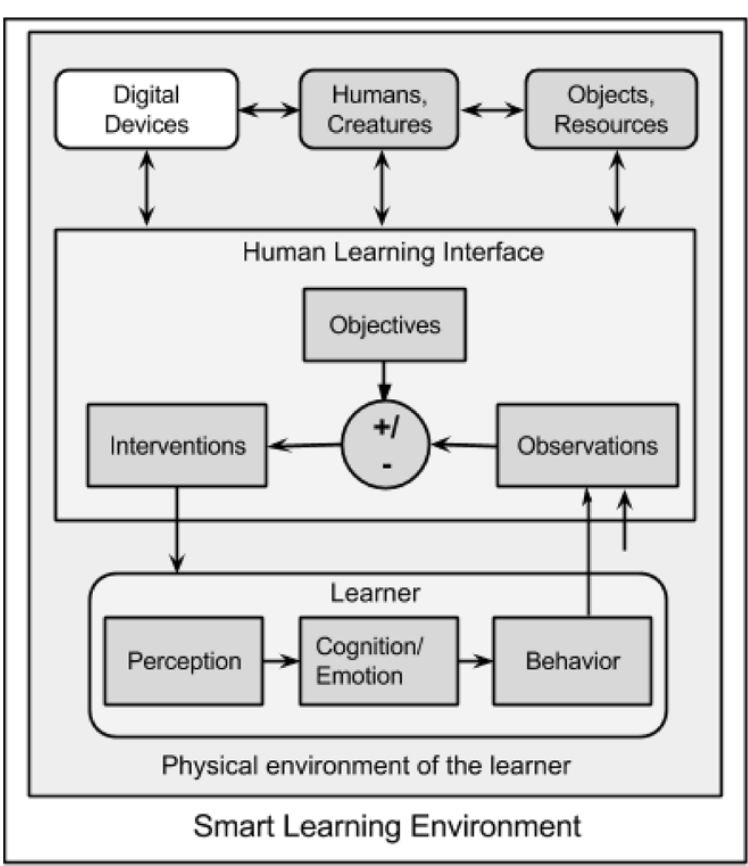

Figure 2 General overview of an human learning interface. 
invokes higher order thinking and testing effects. With respect to testing effects, research has shown that people can better be tested with questions, e.g. self tests, than studying texts in the regular way using mind maps and notes (Carrier and Pashler 1992; Karpicke and Blunt 2011).

2. Give the learner a task to accomplish. Tasks can be very basic ("Look over there, a fox!") to highly complex, such as problems to solve, decisions to make. They can also be an instruction to read or study something ("Read this book").

3. Provide something to the learner. The provisioning of information, an event that occurs in the physical environment, some instruments or other resources that are given to the learner. Provisioning of events, access to persons and tools are part of the arrangement of the learning environment. A learning environment should have the appropriate tools, information and people available in order to facilitate certain learning processes.

4. Conditioning of the environment of the learner. Provide positive and negative feedback, provide incentives, create contingencies, or provide associative stimuli. In most learning environments this is provided by a teacher who praises students for behavior, provide grades, or provide some other forms of incentives. In digital learning environments you also see batches, points and rankings to condition the environment.

The assumption is that more complex instructional interventions can be build from selections and sequences of these core intervention mechanisms. For instance, assessments can be build as combinations of information, tasks, questions and feedback. In SLEs I expect these selections and sequences to be adaptive: the next interventions are dependent on the results of the previous interventions.

\section{Observations}

Observations of a) relevant behaviors, including relevant physical changes and b) relevant changes in the physical environment can be done by humans, or by sensors of digital devices. The observations determine how adaptive an SLE can be. For each of the interventions expected output behaviors should be specified to determine what should be observed. A simple example: a digital device provides the task to look at a painting in a museum and asks the question to identify what the main color pallet is that is used in the painting. Possible behaviors of the learner could include to verbally state a color pallet, to look a bit confused, not know what to answer, to ignore (parts) of the instruction, or to look at something else. In order to provide the correct next intervention, the sensors should be able to observe these states. Observations are also a necessary condition for feedback and assessment. One of the challenges for SLEs is the further development of useful sensors and its use to infer behaviors or changes in the environment.

\section{Objectives}

Specific learning objectives can be set by a user, e.g., a learner or a teacher, of the HLI to evaluate progress towards a defined learning outcome. It is not necessary to set objectives in advance to make the HLI function, it could also consist of a series of 
interventions only, including observations of behavior, but without control. In special cases this could be an approach. Most cases of learning are directed towards certain learning outcomes and need clear objectives to specify these outcomes. For example the taxonomy of Bloom (Bloom et al. 1956; Krathwohl et al. 1964; Anderson et al. 2001) can be used for this purpose.

\section{Different kinds of HLls}

As presented in the paragraph about human learning, different types of learning can be distinguished and for each type I will now define a specific HLI. The categorization I propose is based on the central concept of cognition: representations. What different kinds of representations and related behaviors and learning processes can be distinguished? First of all I will make a distinction between representations of the physical world and representations of the social environment as a subset of the physical world. Representations and related actions of the physical world are needed for adaptation and survival of the individual. "Individuals explore the world and dynamically acquire representations and modify them as needed..." (Sun et al. 2009, p.243). Representations of the affordances and constraints in the social world are needed to adapt to social groups and to carry over cultural learned knowledge and skills between group members and between generations. Members of a species have innate mechanisms to interact with members of the same species and these are distinct from other cognitive processes. For instance people have a very well developed attention mechanisms and memory for human faces and are very sensitive to facial expressions (Hugenberg and Wilson 2013). These two types of representations are related, because the social representations always occur in a physical context. However, learning could be more directed towards the physical world than the social world. Furthermore, in some cases humans can be represented as objects, instead of subjects, e.g. when walking in a busy street. And also, physical objects can be humanized as is done by kids and people who attribute underlying intentions to natural phenomena. All elements of the modal model are expected to be present in both situations, only the type of representation (physical or social) is different.

A next type of learning is based on construction, creation. People are able to create, to design and construct artefacts and new behaviors in interaction with the facilities that are provided in the world. As is stated before, these creative processes involve cognitive re-representations of the world and, because of this, to learning. Another type of learning is directly linked to the encoding and retrieval of representations of knowledge and actions in long term memory. The more something is rehearsed, the more efficient, automatic and faster it becomes. Rehearsal can be seen in two ways (Craik and Lockhart 1972): maintenance rehearsal in which you rehearse to prevent forgetting and elaborative rehearsal to provide better connections with prior knowledge (schema's) in long term memory. This type of rehearsal improves the storage and recall of knowledge, but it can also improve representations of actions in order to perform better and faster. I will refer to this category as practice. Each of the previous three types of learning can be practiced: learning to cope better and faster with physical situations, with social situations and to be able to create something better and faster. The last category I distinguish is the type 2 processing of the central executive in working memory: decision making, planning, overruling experience, and self-regulation. This is about representations of representations (metacognition) and critical reflection of internal representations and 
behavioral impulses. Following this distinction in learning processes, five different HLIs can be identified, each with a different learning goal (Figure 3):

1. Learning to represent new situations and events in the world and know how to act and react. This is facilitated through the identification HLI.

2. Learning to represent the social norms, values, customs and ideologies of social institutions and learning the skills and habits that enables you 'to behave' within the social institutions, including the dissemination of norms and values to others. Social institutions include: family, peer groups, religion, economic system, language and legal system. social situations and events given. This is facilitated through the socialization HLI.

3. Learning to represent new sequences of behavior in order to create something. This is facilitated through the creation HLI.

4. Learning to represent knowledge and actions faster and better, including the representation of performance targets or future incentives for any of the previously mentioned behaviors. This is facilitated through the practice HLI.

5. Learning to create representations of representations and to change the initial representations and behaviors. This is facilitated through the reflection HLI.

In the next paragraphs, each of these HLIs will be discussed.

\section{The identification HLI}

Identification is the learning mechanism that is needed when you encounter a new situation and you learn through experience and acting in the situation. It is like dropping someone in an unknown territory that you have to explore and deal with. The basic learning activities are: exploration, recognition, differentiation and generalization of stimuli, labeling (groups of) stimuli, building knowledge about the behaviors (dangers, contingencies, rules, affordances) of the unknown stimuli, creating mental maps

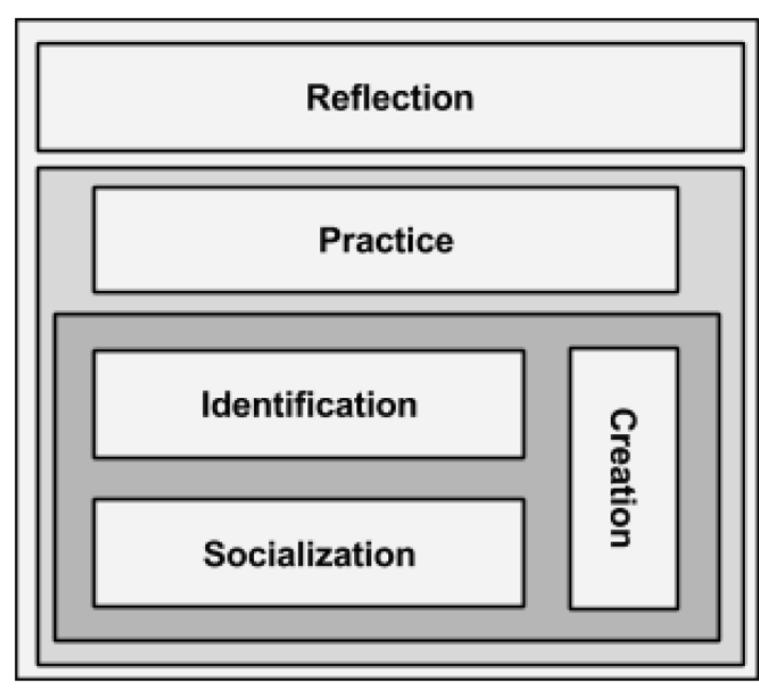

Figure 3 The five HLIs. 
(schema's) of the environment, acting within the situation and solving problems. Some typical learning interventions in this HLI are the following:

- Provide a (simulated or real) situation which is new to the person. The situation includes events and contingencies that challenges the person to react in an appropriate way.

- Give the (implicit or explicit) task to observe and explore the situation and its elements, including possibilities to act and experiment.

- Give the (implicit or explicit) task to identify and categorize differences and commonalities (specialisation and generalization) in a way that the constituents of the situation can be labeled with symbols or names. Ask questions about this and provide feedback.

- Provide stories about the situation, the constituents and its history to learn to know the processes, dynamics, contingencies and characters. A story can be informal, but also a scientifically justified theory. Ask questions about the stories and ask the person to tell stories themselves and map the situation.

- Give the task to perform some activities within the environment, such as travelling, using tools, driving, swimming, etc.

- Provide incentives when conditions are met.

- Give the task to solve some challenging problems and ask "what-if" questions.

Although the list above suggests an order or a pedagogical approach, this is not intended. They should only be seen as possible examples of interventions in the Identification HLI. It is dependent on the concrete setting, the content to be learned, which pedagogical model fits best. Note that the process can be social or nonsocial: exploring new ground with a group or alone. Furthermore, in every step listed above, support can be provided to the person in several ways: by adding a person (teacher; guide; parent; expert) who points at important aspects of the environments and helps to label them ("Look this is an eatable mushroom called champignon") or a digital device with the same functionality. In the context of SLEs this would typically be supported by a digital device or by a combination of a digital device and a supporting person. In all cases the best approach is to scaffold the support: slowly diminish the support to enable the person to act independently (Lave and Wenger 1991).

To create SLEs that use the Identification HLI, it is important to identify prototypical situations that represent the settings that the learner needs to cope with. For instance typical situations that the person has to deal with in a (future) job.

\section{The socialization HLI}

Socialization is the process in which people acquire the cognitive, social and emotional skills that are needed to function in the social community using the social skills for which they are biologically prepared (Bugental 2000). It is for instance needed when travelling to a new country, going to a new class or school, to a new job or having to deal with new customers. Bugental and Grusec (2006) distinguish four types of socialization relationships: attachment relationships that provide proximity between members to maintain safety; social identity relationships to make people feel part of the group and acquire group norms, values and skills; hierarchical relationships that facilitate 
compliance, leadership and discourage maladaptive behaviors; and reciprocal relationships that include the reciprocal exchange of knowledge, benefits and positive affect between peers. They provided evidence that each of these relationships have a different socialization task, developmental course, neurohormonal processes involved and evolutionary support.

Essential learning mechanisms in socialization are observational learning (Bandura 1977) and imitation or mimicry (Chartrand and Van Baaren 2009). Examples of social behaviors that are acquired by observation of others are: self-regulation, aggression, resistance to temptation and moral judgement. People can participate peripherally in societies with the intent to become full members later (Lave and Wenger 1991), during this peripheral participation they are supported to learn the habits and skills of the group by observational learning and supported action. Imitation seems ubiquitous, Dijksterhuis (2013, p.242) states that research has shown that people mimic postures, gestures, facial expressions, and a multitude of speech related phenomena. Interpretation of facial expressions play an important role in socialization. Hugenberg and Wilson (2013, p.167) state that faces can communicate a vast body of information quickly and efficiently, such as facial identities, social categories (e.g., sex, race, age), physical health, evaluations, emotions, and intentions. Facial expressions can provide immediate feedback about the mood of a person or group and can be used to communicate appreciation or disapprovement as a reinforcer for behavior. Some typical learning interventions in the Socialization HLI are the following:

- Provide a (simulated or real) situation in which a person encounters a new social group, people with another cultural background or a variety of customers or people to work with. The situation includes social events, demands and contingencies that challenges the person to react in an appropriate way.

- Give the (implicit or explicit) task to observe the person or group and discover the norms, values, habits and relationships.

- Give the tasks to try to meet and interact with group members, taking their values and habits into account.

- Provide feedback to the person about the activities and answer questions.

- Give the task to mimic activities of others, especially skills and habits that are typical for the group. For example in art groups (dance, painting, music) this is a basic mechanism for learning.

- Give social tasks to execute, such as participating in a specific social and cultural activities, selling a product to a customer, discuss an issue, celebrate something collaboratively, etc.

- Provide incentives when conditions are met.

For SLEs socialization offers the challenge that not only direct communication between actors should be supported, but also indirect communication that mediates the socialization process, especially non-verbal behaviors, including facial expressions. Also effective and non-intrusive sensoring of facial expressions, moods and gestures are needed to fully support socialization in SLEs.

\section{The creation HLI}

Creation is the mechanism in which a person learns by manipulating the environment in such a way that a new object, resource or process is made. What is produced can 
have many forms, such as works of (applied) art, written texts, a meal, a decorated room, a drawing, music, a house, etc. Creation processes involve cognitive change. Creation and technological skills are closely related (Mitcham 1994). Some typical learning interventions in this HLI are the following:

- Provide the (simulated or real) situation and materials that are needed for a creation process.

- Give the task to a person to create something new and provide a framework for incentives for good task accomplishment. The task can be performed alone or in a group.

- Provide feedback to the person about the created artefact and answer questions.

- Give the task to a person to create something, given a set of requirements that must be met, and provide procedures and worked-examples (Sweller 2006).

- Give the task to a person to restructure or decorate an environment.

- Ask questions to the person about the created artefact, e.g. its expression, its use, its quality, how it could be improved.

- Provide incentives when conditions are met.

SLEs should enable to support learning before, during and after the creation process. The creation process itself could take place in the physical world, such as creating a painting on canvas, but can also be supported by a digital device such as a word processor, a paint or design program or even a simulation or game in which you can build structures and processes. In all cases the challenge is that the environment should contain the physical and digital elements that enable the person to learn, including monitoring to adapt interventions.

\section{The practice $\mathrm{HLI}$}

All three of the previously mentioned HLIs, Identification, Socialization and Creation, develop skills that could be further practiced to increase the performance on certain tasks. The core characteristic of practice is to repeat activities to improve the tempo or the quality of the result and to prepare for a high performance in future situations. Practice of skills also leads to automation of these skills. Practice could be performed in reality, but also in play. Play is a way of practice that can been seen in all mammals and other species such as birds. Play has no sense in itself, but prepares the person to cope with future real situations. People can play alone or with peers and represent mentally (parts of) the environment in which the activities are set (the playground), including rules and performance criteria. Important aspects of practice and play are: a) there should be a suitable (real or partly imaginary) environment, including tools and peers for the activities to be performed, b) there should be the possibility to repeat the same activity over and over again, c) performance measures and standards should be set, along with incentives, d) feedback about the accomplishments per repetition should be made available and e) incentives provided. Examples of learning environments that use the practice HLI are classical drill and practice programs and serious games. But in fact, all learning environments that support repetition and performance criteria are suitable as a practice environment. In smart learning environments the practice devices are expected to be context-aware and adaptive, e.g. like GPS sport watches could be used 
as a digital feedback device to learn to perform better. The practice HLI is a meta-HLI and envisaged to be integrated into each of the three previous HLIs.

\section{The reflection HLI}

The reflection HLI stimulation of system 2 ('hard') thinking: reflection, reframing of the problem and solution, evaluation of results, decision making, strategy development, self-regulation, and non-monotonic, deep learning as described by Ohlsson (2011). This involves overruling previous experience, trying out new ideas, creative insight, adaptation of cognitive skills by learning from errors, and conversion from one believe to another incompatible believe. Reflection can be stimulated as follows: a) stimulate people to take the time to consider different options before they act; b) stimulate people in creative thinking, coming up with new ideas and solutions, c) providing the possibility to review and analyse their actions and effects and reflect on possible improvements, d) provide possibilities to test yourself by asking questions, evaluate the objectives, environment and behavior, e) stimulate the decision making processes, and facilitate strategy development. The reflection HLI is also a meta HLI to all four previously mentioned HLIs. This means that a smart learning environment that is focussed on Identification, this learning environment should also include facilities for practice and for reflection in order to stimulate faster and better learning. The same is true for Socialization and Creation.

\section{Conditions for smart learning environments Definition}

Taking this all together, the concept of SLEs can now be defined as follows: SLEs are physical environments that are improved to promote better and faster learning by enriching the environment with context-aware and adaptive digital devices that, together with the existing constituents of the physical environment, provide the situations, events, interventions and observations needed to stimulate a person to learn to know and deal with situations (identification), to socialize with the group, to create artefacts, and to practice and reflect.

It should be noted that the HLIs are defined independent of any specific content domain, any specific pedagogical or instructional approach or any specific technologies in mind. This makes the approach generic applicable as a theoretical model for all types of smart learning environments. In practice of course, these specific choices should be made before an SLE can be implemented and used. A second note is that I have focussed on the core objective of SLEs, better and faster learning, but besides that core objective, SLEs also need to be safe, reliable, inclusive and engaging. These latter aspects are not in scope within this paper, but also need attention and further elaboration.

\section{Conditions}

To summarize the conditions for the development of effective SLEs:

1. An SLE is a learning environment in which one or more digital devices are added to the physical locations of the learner to add learning functions, to add virtual or augmented situations \& locations, to add facilities for the monitoring and assessment of the progress of learners and to add facilities for the provision of appropriate information to relevant stakeholders; 
2. With respect to the learning functions, the SLE supports at least one of the three core HLI's: identification, socialization and creation, depending on the objectives to be attained with the SLE.

3. The SLE supports both meta HLI's in order to stimulate practice and reflection on the core HLI's.

4. The SLE implements the functionality to observe the learners location, context, preferences, physical and mental condition and culture and adapts interventions based on relevant observations.

5. The SLE implements the intervention mechanisms: questions, tasks, provision of information \& resources, and conditioning.

6. The SLE implements functionality to specify and communicate learning objectives.

7. The processes in the SLE do not cause frictions, like waiting times and other inefficiencies. The processes includes enrollment, teaching and learning, examination and support and information facilities.

\section{Discussion and conclusion: a research agenda for SLEs}

As stated in the introduction, smart(er) learning environments promote better and faster learning. In order to do so, I presented the idea of Human Learning Interfaces as the set of interaction mechanisms that humans expose, and that can be used to control, stimulate and facilitate their learning processes. I distinguished three basic HLIs (identification, socialization and creation) and two meta HLIs (practice and reflection), of which reflection represents higher-order thinking of type 2 as it is seen in dual process theory of cognition. Based on this analysis the conditions for the development of Smart Learning Environments are defined.

What future research is needed in order to develop SLEs that are utilizing the HLIs effectively? I will now present a provisional research agenda in order to realize this ambition of SLEs. Some of this research is more oriented at cognition, learning and instruction and some more at the technological aspects of SLEs:

1) Which sequences of interventions stimulate faster and better learning in each of the three core HLIs? The objective is to provide clear prescriptions how to arrange interventions to help people to learn faster and better. The same question should be asked for the meta-HLIs (practice and reflection). It could be expected that the meta-HLI's have some generic functions, but also specific functions per core HLI. Furthermore, the HLIs should be more formalized to represent an interface description that provides exact requirements for the developers of SLEs, including a testing framework.

2) Which individual factors and differences are of importance for the adaptation of interventions within SLEs? People differ on many dimensions and SLEs should adapt to relevant differences. The first thing to consider are the differences in the preferences of persons, i.e. preferences in 'what' to learn and 'how' to learn. For instance, can these preferences, especially about 'how to learn', be summarized in multidimensional preference profiles that are relevant for the selection of interventions? Another aspect to take into account are differences in the physical and mental condition, the motivation, concentration, self-efficacy and the personal situation of the individual. Which of these differences are relevant triggers for 
adaptations of interventions? It should be noted that individual differences research is hard, because many attempts have failed to create impact (e.g. ATI research and research on learning styles of Pashler et al. 2008). Adaptive systems have at the moment very weak grounded effects on learning; more fundamental research into individual differences and the adaptation of interventions towards these differences is required in order to create more effective adaptive systems.

3) Which reactions of a person - as a consequence of an intervention - requires an adaptation of the subsequent interventions and how are these reactions observed? The observations can be formative or summative assessments of learning outcomes, but also of responses to interventions: interventions do not always invoke the expected reaction, e.g. people can be disturbed, have a different understanding or provide a response that is inadequate. In a learning environment, most of these observations are normally done by humans, e.g. teachers and peers. It would be interesting to research whether (parts) of these observations can also be automated by digital devices with adequate sensors. Part of this question is research which adaptations of interventions are effective for each HLI based on observations of individual responses, preferences, conditions, locations, context and culture? This is a core question that can only be answered after answering the previous questions. An important point to note is the term 'needed'. Many adaptation rules can be implemented, but are they really required to improve learning?

4) Which factors in the organization of the processes within the SLE (enrollment, teaching and learning, examination, support and information) and which external factors can disturb a smooth flow through the process for learners, teachers and others, i.e. cause frictions, dissatisfaction and dropout? How can this be improved? Examples are the inclusion of unnecessary waiting times, enrollment constraints, grouping of students, inefficient temporal organization of the curriculum, the degree of flexibility in tempo, etc.

5) How can we arrange SLEs that convincingly improve learning in comparison to existing learning environments. Stated differently: how to develop SLEs or adapt existing learning environments that meet the conditions for effective SLEs? Experiments are needed, a catalogue of best practices, and also reference architectures and other guidelines for the development of effective SLEs.

6) And last but not least, more fundamental research is also needed to the HLIs themselves, for instance are they distinct categories at the neuro-cognitive level, or mere interfaces that work on top of generic processes? Are there differences between persons in the functioning of the HLIs? How do they develop over time? Do the three core HLIs interact or are they distinct? Can they be trained?

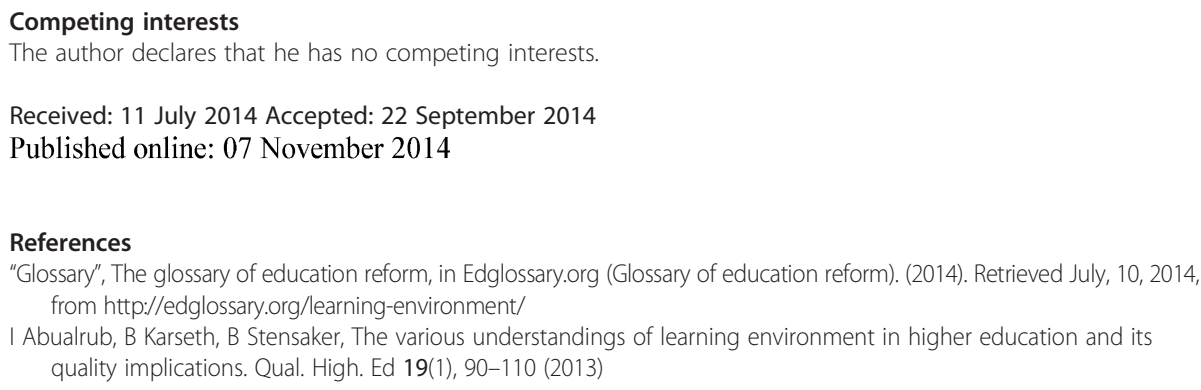


LW Anderson, DR Krathwohls, PW Airasian, KA Cruikshank, RE Mayer, PR Pintrich, J Raths, MC Wittrock, A Taxonomy for Learning, Teaching, and Assessing: A Revision of Bloom's Taxonomy of Educational Objectives (complete ed.) (Longman, New York, 2001)

MH Ashcraft, GA Radvansky, Cognition, 5th edn. (Pearson, NJ, 2010)

RC Atkinson, RM Shiffrin, Human memory: a proposed system and its control processes. Psychol. Learn. Motiv. 2, 89-195 (1968)

RC Atkinson, RM Shiffrin, The control processes of short-term memory. Sci. Am. 225, $82-90$ (1971)

A Bandura, Social Learning Theory (Prentice Hall, Englewood Cliffs NJ, 1977)

A Bandura, Social cognitive theory of self-regulation. Organ. Behav. Hum. Decis. Process. 50(2), 248-287 (1991)

A Bandura, RW Jeffrey, Role of symbolic coding and rehearsal processes in observational learning. J. Pers. Soc. Psychol. 26(1), 122 (1973)

A Bandura, D Ross, SA Ross, Transmission of aggression through the imitation of aggressive models. J. Abnorm. Soc. Psychol. 63(3), 575-582 (1961)

A Bandura, D Ross, SA Ross, Imitation of film-mediated aggressive models. J. Abnorm. Soc. Psychol. 66(1), 3-11 (1963)

RF Baumeister, E Bratslavsky, M Muraven, DM Tice, Ego depletion: Is the active self a limited resource? J. Pers. Soc Psychol. 74(5), 1252-1265 (1998)

BS Bloom, MD Engelhart, EJ Furst, WH Hill, DR Krathwohl, Taxonomy of Educational Objectives: The Classification of Educational Goals (handbook l: cognitive domain) (David McKay Company, New York, 1956)

JS Brown, A Collins, AP Duguid, Situated cognition and the culture of learning. Educ. Res. 18(1), 32-42 (1989)

DB Bugental, Acquisition of the algorithms of social life: a domain-based approach. Psychol. Bull. 26, 187-209 (2000)

DB Bugental, JE Grusec, Socialization processes, in Handbook of Child Psychology: vol. 3. Social, Emotional, And Personality Development, ed. by W Damon, N Eisenberg, 5th edn. (Wiley, New York, 2006), pp. 366-428

DL Butler, PH Winne, Feedback and self-regulated learning: a theoretical synthesis. Rev. Ed. Res. 65(3), 245-281 (1995)

DE Carlston, The Oxford Handbook of Social Cognition (Oxford Press, Oxford, 2013)

M Carrier, H Pashler, The influence of retrieval on retention. Mem. Cognit. 20, 632-642 (1992)

TL Chartrand, R Van Baaren, Human mimicry. Adv. Exp. Soc. Psycol. 41, 219-274 (2009)

FI Craik, RS Lockhart, Levels of processing: a framework for memory research. J. Verbal. Learn. Verbal. Behav. 11(6), 671-684 (1972)

A Dijksterhuis, Automaticity. Handbook of social cognition, 2013, pp. 239-256

JSBT Evans, How many dual-process theories do we need? One, two or many? in Two Minds: Dual Processes and Beyond, ed. by JSBT Evans, K Frankish (Oxford Un. Press, Oxford, 2009), pp. 33-54

JSBT Evans, DE Over, Rationality and Reasoning (Psychology Press, Hove, 1996)

EA Griffin, A First Look at Communication Theory, 8th edn. (McGraw-Hill, Boston, 2012)

T Gross, M Specht, Awareness in context-aware information systems. Mensch \& Computer 2001 Berichte des German Chapter of the ACM 55, 173-182 (2001)

FW Hafferty, JP Hafler, The hidden curriculum, structural disconnects, and the socialization of new professionals. Innov. Change. Prof. Educ. 6, 17-35 (2011)

E Higgins, WS Rholes, CR Jones, Category accessibility and impression formation. J. Exp. Soc. Psychol. 13(2), 141-154 (1977)

K Hugenberg, JP Wilson, Faces are central to social cognition, in Handbook of Social Cognition (2013), pp. 167-193

D Kahneman, S Frederick, A model of heuristic judgment, in The Cambridge Handbook of Thinking and Reasoning, ed. by K Holyoak, RG Morrison (Cambridge University Press, Cambridge, 2005), pp. 267-294

D Kahneman, A Tversky, Prospect theory: an analysis of decision under risk. Econometrica. J. Econometric. Soc. 47(2), 263-291 (1979)

JD Karpicke, JR Blunt, Retrieval practice produces more learning than elaborative studying with concept mapping. Science 331, 772-775 (2011)

DR Krathwohl, BS Bloom, BB Masia, Taxonomy of Educational Objectives: The Classification of Educational Goals. Handbook II: the Affective Domain (David McKay Company, New York, 1964)

J Lave, E Wenger, Situated Learning: Legitimate Peripheral Participation (Cambridge University Press, Cambridge, 1991)

P Mayer, Socialization: the Approach from Social Anthropology (first print 1970) (Routledge, London, 2013)

MD Merrill, First principles of instruction. Educ. Technology. Res. Dev. 50(3), 43-59 (2002)

DE Meyer, RW Schvaneveldt, Facilitation in recognizing pairs of words: evidence of a dependence between retrieval operations. J. Exp. Psychol. 90, 227-234 (1971)

C Mitcham, Thinking Through Technology (The University of Chicago Press, Chicago, 1994)

S Ohlsson, Deep Learning: How The Mind Overrides Experience (Cambridge University Press, Cambridge, 2011)

H Pashler, M McDaniel, D Rohrer, R Bjork, Learning styles concepts and evidence. Psychol. Sci. Publ. Interest. 9(3), 105-119 (2008)

D Schunk, Learning Theories: An Educational Perspective. Learning theories: An educational perspective (6th edit) (Pearson Education Inc, Boston, 2012)

SA Sloman, The empirical case for two systems of reasoning. Psychol. Bull. 119(1), 3 (1996)

KE Stanovich, Who is Rational?: Studies of Individual Differences in Reasoning (Lawrence Elrbaum Ass, Mahwah NJ, 1999)

KE Stanovich, The Robot's Rebellion: Finding Meaning in the Age of Darwin (University of (Chicago Press, Chicago, 2004)

R Sun, SM Lane, RC Mathews, The two systems of learning: An architectural perspective, in Two Minds: Dual Processes and Beyond, ed. by JSBT Evans, K Frankish (Oxford University Press, Oxford, 2009), pp. 239-264

J Sweller, The worked example effect and human cognition. Learn. Instruct. 16(2), 165-169 (2006)

A Tversky, D Kahneman, Judgment under uncertainty: heuristics and biases. Science 185(4157), 1124-1131 (1974)

LS Vygotsky, Mind in Society: The Development of Higher Psychological Processes (Harvard University Press, Harvard, 1978)

doi:10.1186/s40561-014-0005-4

Cite this article as: Koper: Conditions for effective smart learning environments. Smart Learning Environments 2014 1:5. 